Draft VERSiOn MARCh 29, 2018

Preprint typeset using $\mathrm{AT}_{\mathrm{E}} \mathrm{X}$ style emulateapj v. 5/2/11

\title{
FORMING THE COLD CLASSICAL KUIPER BELT IN A LIGHT DISK
}

\author{
Andrew Shannon ${ }^{1,3}$, YANQIn $\mathrm{WU}^{1} \&$ Yoram Lithwick ${ }^{2}$ \\ ${ }^{1}$ Department of Astronomy and Astrophysics, University of Toronto, Toronto, ON M5S 3H4, Canada; \\ ${ }^{2}$ Department of Physics and Astronomy, Northwestern University, Evanston, IL 60208 and Center for Interdisciplinary Exploration and \\ Research in Astrophysics (CIERA) and \\ ${ }^{3}$ Institute of Astronomy, University of Cambridge, Madingley Road, Cambridge CB3 0HA, UK \\ Draft version March 29, 2018
}

\begin{abstract}
Large Kuiper Belt Objects are conventionally thought to have formed out of a massive planetesimal belt that is a few thousand times its current mass. Such a picture, however, is incompatible with multiple lines of evidence. Here, we present a new model for the conglomeration of Cold Classical Kuiper belt objects, out of a solid belt only a few times its current mass, or a few percent of the solid density in a Minimum Mass Solar Nebula. This is made possible by depositing most of the primordial mass in grains of size centimetre or smaller. These grains collide frequently and maintain a dynamically cold belt out of which large bodies grow efficiently: an order-unity fraction of the solid mass can be converted into large bodies, in contrast to the $\sim 10^{-3}$ efficiency in conventional models. Such a light belt may represent the true outer edge of the Solar system, and it may have effectively halted the outward migration of Neptune. In addition to the high efficiency, our model can also produce a mass spectrum that peaks at an intermediate size, similar to the observed Cold Classicals, if one includes the effect of cratering collisions. In particular, the observed power-law break observed at $\sim 30 \mathrm{~km}$ for Cold Classicals, one that has been interpreted as a result of collisional erosion, may be primordial in origin.
\end{abstract}

Subject headings: Numerical simulations: Planetesimal growth

\section{INTRODUCTION}

Beyond Neptune lies the Kuiper Belt, a population of remnant planetesimals that were never incorporated into planets (Edgeworth 1949; Jewitt \& Luu 1993; for a recent review, see Barucci et al. 2008). Of particular interest here is the Cold Classical Kuiper Belt, a population of low-inclination $\left(i<5^{\circ}\right)$, low eccentricity objects lying between 42 and $47 \mathrm{AU}$ (Brown 2001; Trujillo \& Brown 2002; Elliot et al. 2005) with a total mass of $\sim 0.1 M_{\oplus}$ (Fuentes \& Holman 2008; Fraser \& Kavelaars 2009; Vitense et al. 2010) 1 and sizes up to $\sim 200 \mathrm{~km}$ in radius (Levison \& Stern 2001; Fraser et al. 2010).

While the other Kuiper belt populations (the resonant and the scattered bodies) appear to have been injected into the region via interactions with planets (Malhotra 1993, 1995; Luu et al. 1997; Gomes et al. 2008), the quiescent orbits of the Cold Classical population cannot be produced that way (Levison et al. 2008; Dawson \& Murray-Clay 2012), nor would their delicate long-period binaries have survived such havoc (Parker \& Kavelaars 2010). The evidences strongly favour an in situ formation of the Cold Classical Kuiper Belt (although for a dissenting view, see Morbidelli et al. 2009, 2014). The color and size distributions of the Cold Classicals differ markedly from the other populations (Tegler \& Romanishin 2000; Levison \& Stern 2001; Brown 2001; Noll et al. 2008; Brucker et al.|2009; Fraser et al.|2010, 2014; Perna et al. 2010; Fraser \& Brown 2011; Lacerda et al.|2014), further cementing its different origin.

1 This estimate is uncertain by a factor of a few, affected by the assumed albedo, among other things.
The in-situ formation of the Cold Classicals, beyond the dynamical ravage of the giant planets, allows us to place strong constraints on their formation history. This gives hope that one can piece together the giant puzzle, the origin of the outer solar system bodies, as well as bodies in other outer planetary systems (manifested as extra-solar debris disks), by scrutinizing this unique population.

\subsection{Formation in a heavy disk-problems}

Previous studies for the formation of Kuiper belt bodies focussed on their conglomeration out of a sea of kilometer-sized seeds, perhaps instigated by the planetesimal formation scenario of Safronov (1969) and Goldreich \& Ward (1973). Pairwise collisions between these seeds produce large enough bodies that subsequently grow by accreting the remaining seeds. Meanwhile, the $\mathrm{km}$-sized bodies are viscously stirred by these growing big bodies. The higher velocity dispersion allows bigger bodies to grow more rapidly than smaller bodies, quickly leaving them behind, in a process termed runaway growth Greenberg et al. 1978; Wetherill \& Stewart 1989). Eventually, the runaway bodies stir the $\mathrm{km}$ bodies faster than they can accrete them (Kenvon \& Bromley 2008; Ormel et al. 2010). Growth stalls.

In these models, growth lasts for a few collisional times of the km-sized seeds, and it stalls when the biggest bodies have reached of order Pluto size $\left(\sim 10^{3} \mathrm{~km}\right)$. There is little evolution in either the size spectrum or the total mass of large Kuiper belt objects in the billions of years that follow, as the accretion time becomes longer than the system lifetime (Kenyon \& Luu 1999). In these models, the km-sized seeds collide much less rapidly than 
they are stirred up. Collisional cooling is unimportant for the dynamics, and we name this scenario 'collisionless growth'.

Two generic features are observed in all simulations of collisionless growth (Greenberg et al. 1978; Wetherill \& Stewart 1989; Weidenschilling et al. 1997; Kenvon \& Luu 1998; Kenvon \& Bromley 2008: Ormel et al. 2010; Schlichting \& Sari 2011). The efficiency of forming big bodies, defined as the fraction of the total initial mass that resides in bodies much larger than the seeds, is generically $\sim 10^{-3}$ at the Kuiper belt distance (Schlichting \& Sari 2011; Shannon et al. 2015). The differential mass distribution of these big bodies

$$
\frac{d M}{d \log R} \propto R^{n},
$$

is flat for each logarithmic size decade, or $n=0$ These two features are simply understood using the analytical arguments in Lithwick (2014): they arise because during conglomeration, the largest bodies regulate their own growth by exciting the dispersion of small bodies to of order their own Hill velocity, or, small bodies remain roughly 'trans-hill' relative to the largest bodies at any moment. Using our newly developed conglomeration code, we have carefully tested this analytical understanding (Shannon et al. 2015).

For a formation efficiency of $\sim 10^{-3}$, a total mass in large Cold Classical bodies of $\sim 0.1 M_{\oplus}$ will demand a primordial solid mass of $\sim 100 M_{\oplus}$, comparable to the solid mass expected for the Kuiper belt region in a Minimum Mass Solar Nebula (MMSN Weidenschilling 1977b; Havashi 1981). However, such a set-up appears to be excluded by multiple lines of evidence:

- Models for the migration of Neptune find that the primordial MMSN disk must end around 30 AU, or Neptune would have continued its outward march (Fernandez \& Ip 1984; Thommes et al. 1999; Gomes et al. 2004).

- If there had been $\gtrsim 1 M_{\oplus}$ of solids in km-sized bodies in the Kuiper Belt, long period Kuiper belt binaries would have been disrupted by impacts (Parker \& Kavelaars 2012; Parker et al. 2011).

- If the efficiency of forming the Cold Classical is low, $99.9 \%$ of the primordial mass (stored in small bodies like $1 \mathrm{~km}$ planetesimals) has to be lost by collisional grind-down, followed by radiation pressure blow-out or Poynting-Robertson drag. However, attempts to model this loss process typically do not remove more than $\sim 90 \%$ of the mass (Kenyon \& Bromlev 2004), even in the most optimistic models (Pan \& Sari 2005).

- If one invokes dynamical ejection by giant planets to remove the primordial mass, disregarding the fact that the Cold Classicals are detached from the giant planets, since dynamical ejection has the attribute of removing all body sizes equitably, this would have required an even higher primordial mass.

2 The more commonly used differential size distribution, $d N / d R \propto R^{-q}$ is related as $q=4-n$.
We conclude that the low formation efficiency, characteristic of collisionless conglomeration, is not astrophysically viable for the Cold Classicals.

The last line of argument also applies to the hot Kuiper belt objects (the scattered and the resonant populations): if they were formed with a similarly low efficiency, then Neptune, during its outward migration, would have to carefully preserve these precious products, or else the primordial disk has to be much more massive than the corresponding MMSN value. Lastly, old extra-solar debris disks, comparable in age to the Solar System yet with dust luminosity three or more orders of magnitude above that in the Kuiper belt (Bryden et al. 2006), require $\sim 10 M_{\oplus}$ in the form of large bodies (Shannon \& Wu 2011). This is also incompatible with the low efficiency of collisionless growth.

So the presence of Cold Classicals, and perhaps also the hot Kuiper belt objects and extra-solar debris disks, call for a new formation scenario.

\subsection{Formation in a light disk}

In this work, we explore a new model of formation, where the large KBOs we observe today were formed in a proto-Kuiper belt that is light in mass and contained mostly centimeter-sized grains, along with some kilometer-sized seed planetesimals. This approach is motivated by the Goldreich et al. (2004) proposal where they introduced small grains to shorten the formation timescale for Neptune and Uranus. Dynamic cooling by collisions among the centimeter sized grains reduces their random velocities, and fundamentally alters the dynamics. The encounter velocities between bodies are, in this case, dictated by the Keplerian shear (the so-called 'shear-dominated' regime), rather than by intrinsic velocity dispersion. In this regime, runaway growth is avoided, and large bodies grow until a formation efficiency of order unity is achieved.

The now much higher efficiency permits the in situ formation of the Cold Classicals, within a few million years. The primordial belt only needs to have a mass of $\sim 0.1 M_{\oplus}$, comparable to the mass in large KBOs today, and orders of magnitudes lower than the MMSN value. This circumvents the pitfalls in models of collisionless growth. We call such a primordial disk the 'Minimum Mass Kuiper Belt' (MMKB). The size spectrum from such a 'collisional growth' may be made compatible with the observed KBOs.

Are we justified in adopting such an initial condition, with kilometre-sized rocks swimming in swarms of centimetre grains?

Dust sticking within the protoplanetary disk may have converted much of the microscopic grains to larger particles (see review by Blum \& Wurm 2008). This growth, however, may be sabotaged by collisional fragmentation when the cm-range is reached (Brauer et al. 2008), or arrested by bouncing, again at centimeter sizes (Zsom et al. 2010). This motivates us to choose centimetre for our small bodies. In addition, observations of comet $103 \mathrm{P} /$ Hartley 2 show that much of its dusty coma is made up of grains $1-10 \mathrm{~cm}$ in size (Kellev et al. 2013, and its erratum), which may represent the material it formed from (Kretke \& Levison 2015). Lastly, centimeter-sized grains are observed to persist at tens of au for millions of years in proto- 
planetary disks (e.g., Pérez et al. 2012; Isella et al. 2012; Trotta et al. 2013). This runs against the prevailing argument that aerodynamic drag, exerted by a gaseous disk on these grains, should bring them inward rapidly (Weidenschilling 1977a), and points to missing ingredients in our understanding of their evolution.

A population of large seeds is necessary for our model. The gravitational potential of these bodies can accrete and retain small bodies, so growth does not have to reply on particle sticking, which may be difficult beyond the cm-size (Blum \& Wurm 2008). These bodies should be of order kilometre or larger to allow rapid accretion in the initial stage, but their exact size and mass fraction do not impact our results (see 84.3 ). A recent investigation of Comet $67 \mathrm{P}$ indicates that it is likely a result of gentle collision between two kilometresized bodies (Massironi \& Simioni 2015). This suggests that such bodies may be common in the Kuiper belt region during the early stage. Coincidentally, in a low-mass protoplanetary disk as the one assumed here, if the grains settle and concentrate toward the disk midplane and undergo gravitational instability (Toomre 1964; Goldreich \& Ward 1973), the solid mass that lies within a Toomre wavelength corresponds to that of a kilometre body. 3

So in this work, we assume that by the time gas disk dissipates, one may be left with a host of km-sized planetesimals, surrounded by a sea of cm-grains. These assumptions are critical to our results, but as is clear from the above discussion, while multiple pieces of circumstantial evidence suggest that such a set of initial conditions may be realistic, we are far from being able to prove it based on first-principle.

In spirit, our conglomeration model is akin to the "pebble accretion' model proposed for the formation of planetary cores in gaseous disks (e.g. Lambrechts \& Johansen 2012; Kretke \& Levison 2015). While in that model, gas drag assists cm-sized 'pebble' to be accreted onto large seeds (Rafikov 2003b; Ormel \& Klahr 2010), pebbles here are cooled dynamically by mutual collision, leading to efficient accretion onto large seeds.

This paper is arranged as follows. We present the theory and simulations for collisionally-cooled growth in $\$ 2$ \& 33, respectively. We then discuss the observational implications of our results (\$4), including comparing our predicted size spectrum against the observed one, and discuss alternative formation models.

\section{COLLISIONAL GROWTH: THEORY}

In the following subsections, we work out how collisionally-cooled accretion proceeds, and derive the efficiency of formation. The readers are referred to Lithwick (2014) for a more general discussion that includes both collisional and collisionless conglomeration. Here, we assume that small body size is constant during the evolution.

\subsection{Prelude: Symbols and Values}

${ }^{3}$ Goldreich \& Ward (1973); Goldreich et al. (2004) have argued that gravitational instability only form much smaller bodies as the angular momentum contained in a Toomre wavelength is too large to allow direct collapse into solid density. However, this is not seen in simulations of Michikoshi et al. (2007, 2009).
We adopt formulae for accretion, viscous stirring, collision, and accretion as summarized by Goldreich et al. $(2004)$, along with their notation: big bodies have radius $R$, velocity dispersion $v$, and surface density $\Sigma$; small bodies have radius $s$, velocity dispersion $u$, and surface density $\sigma$; all bodies have bulk density $\rho$ which we take to be $\rho=1.5 \mathrm{~g} / \mathrm{cm}^{3}$, same as the Sun.

We define an important dimensionless quantity

$$
\alpha \equiv R / R_{\mathrm{H}} \sim R_{\odot} / a
$$

which is the ratio of the physical size of a body to its Hill sphere, or, approximately, the apparent angular size of the Sun at distance $a\left(R_{\odot} / a\right.$, where $R_{\odot}$ is the solar radius). For the Cold Classical region, $a \sim 45 \mathrm{AU}$, so $\alpha \sim 10^{-4}$.

In the Kuiper belt, the orbital frequency is $\Omega \sim$ $0.02 / \mathrm{yr}$. The Hill velocity for a body of size $R$ is,

$$
v_{H} \sim \Omega a\left(\frac{R}{R_{\odot}}\right) \sim 30 \mathrm{~cm} / \mathrm{s}\left(\frac{R}{100 \mathrm{~km}}\right) .
$$

The escape speed from the surface of such a body is $v_{\text {esc }} \sim \alpha^{-1 / 2} v_{H} \sim 100 v_{H}$.

If we spread a mass of $0.1 M_{\oplus}$ uniformly within an annuli between 42 and $48 \mathrm{AU}$, we obtain a surface density $\sigma=\sigma_{\mathrm{MMKB}} \approx 0.0016 \mathrm{~g} / \mathrm{cm}^{2}$, where the subscript MMKB denotes 'minimum mass Kuiper Belt'.

In such a disk, collision time between grains is short,

$$
t_{\mathrm{col}} \sim \frac{\rho s}{\sigma \Omega} \sim 0.03 \mathrm{Myr}\left(\frac{s}{1 \mathrm{~cm}}\right)\left(\frac{\sigma}{\sigma_{\mathrm{MMKB}}}\right)^{-1} .
$$

During the growth, we assume that the relevant velocity dispersions, that of big bodies that dominate viscous stirring (bodies with the highest $\Sigma(R)$ ), and that of small grains, are both sub-hill relative to the stirrer.

$$
\begin{array}{r}
v<v_{H}, \\
\alpha^{1 / 2} v_{H}<u<v_{H} .
\end{array}
$$

So accretion is always in the sub-hill, orderly growth regime. This ensures high efficiency of formation. We verify these assumptions here.

During growth, velocity dispersion of small bodies reaches a quasi-equilibrium, balancing collisional cooling among them and viscous stirring by big bodies,

$$
\frac{1}{u} \frac{d u}{d t} \sim \frac{\Sigma \Omega}{\rho R} \alpha^{-2} \frac{v_{H}}{u}-\frac{\sigma \Omega}{\rho s} \approx 0,
$$

or,

$$
\frac{u}{v_{H}} \sim \frac{\Sigma}{\sigma} \frac{s}{R} \alpha^{-2}
$$

So the sub-hill condition $\left(u<v_{H}\right)$ is satisfied if $s$ is sufficiently small,

$$
s \leq \frac{\sigma}{\Sigma} R \alpha^{2} .
$$

We verify that this is satisfied throughout our simulation.

Velocity dispersion of the big bodies, on the other hand, is determined by the balance between self-stirring and dynamical friction from small bodies,

$$
\frac{1}{v} \frac{d v}{d t} \sim \frac{\Omega}{\rho R} \alpha^{-2}\left(\Sigma \frac{v_{H}}{v}-\sigma\right) \approx 0 .
$$


Hence,

$$
\frac{v}{v_{H}} \sim \frac{\Sigma}{\sigma} .
$$

So until unity efficiency is reached, big bodies remain sub-hill, consistent with inequality (5).

\subsection{Growth under Equal Accretion}

Under eq. (6), growth of big bodies by accreting small bodies proceeds at a rate (Goldreich et al. 2004),

$$
\left.\frac{1}{R} \frac{d R}{d t}\right|_{\text {small }} \sim \frac{\sigma \Omega}{\rho R} \alpha^{-1}\left(\frac{v_{\mathrm{H}}}{u}\right) \sim \frac{\sigma^{2} \Omega}{\Sigma \rho s} \alpha
$$

While accretion of small bodies dominates growth at early stages, accretion of big bodies of comparable sizes becomes increasingly important over time. We find that the growth naturally enters into the so-called 'equal accretion' phase where the two rates become comparable.

Big bodies grow by accreting one another at the rate

$$
\left.\frac{1}{R} \frac{d R}{d t}\right|_{\text {big }} \sim \frac{\Sigma \Omega}{\rho R} \alpha^{-3 / 2} .
$$

Note that this rate is independent of $v$, whereas eq. (12) is $\propto 1 / u$. The rates differ because sub-Hill big bodies lie in a flat disk, whereas small ones have an isotropic velocity dispersion due to collisions (Ida \& Makino 1992; Rafikov 2003a; Goldreich et al. 2004).

Defining the ratio between two modes of accretion to be

$$
f \equiv \frac{d \ln R /\left.d t\right|_{\text {small }}}{d \ln R /\left.d t\right|_{\text {big }}} \sim\left(\frac{\sigma}{\Sigma}\right)^{2}\left(\frac{R}{s}\right) \alpha^{5 / 2},
$$

we find that the system naturally tends toward $f \approx 1$. If $f \gg 1$, as is the initial condition in our simulations, small body dominates accretion and both $\Sigma$ and $R$ rise. However, the rise of $\Sigma$ is faster since mass scales as $R^{3}$. This reduces $f$ toward unity. On the other hand, if $f \ll 1$ to start with, big bodies grow by accreting each other. This increases $R$ while keeping $\Sigma$ constant, thereby boosting $f$ toward unity. We conclude that

$$
f \rightarrow 1
$$

at late times, a phase we call the 'equal accretion' phase. As a result, the fraction of mass in big bodies increases with $R$ as

$$
\frac{\Sigma}{\sigma} \sim \sqrt{\frac{R}{s}} \alpha^{5 / 4} .
$$

This is one of the central results of this paper. The formation efficiency can reach unity for a sufficiently large $R / s$. We need small bodies to grow large bodies efficiently.

Equal accretion was first suggested by Schlichting \& Sari (2011), but they considered the collisionless case only. In Shannon et al. (2015), we showed that in the collisionless case, equal accretion is a consequence of the dynamics, rather than the driving factor that it is in the collisional case.

Comparing eqs. (9) and (16), it appears that the equal accretion phase can only commence after

$$
R \geq s \alpha^{-3 / 2}
$$

or $R \gtrsim 10 \mathrm{~km}(\mathrm{~s} / 1 \mathrm{~cm})$ for the Kuiper Belt 4

Moreover, we verify that our assumption on small body dispersion, en. (6), is satisfied during equal accretion. Combining eqs. (8) and (16), one obtains

$$
\frac{u}{v_{H}} \sim \sqrt{\frac{s \alpha^{-3 / 2}}{R}}
$$

Therefore, the ratio $u / v_{H} \sim 1$ when equal accretion begins, and it falls to $u / v_{H} \sim \sqrt{\alpha}$ at its completion $(\Sigma \sim \sigma)$. The value of $u$ itself rises with time (assuming $s$ is constant) as $u \propto R^{1 / 2}$.

\subsection{End of Equal Accretion}

If equal accretion proceeds indefinitely, eq. (16) implies that accretion reaches a radius of completion (i.e. unity efficiency) at

$$
R \sim s \alpha^{-5 / 2}
$$

For the Cold Classical belt, this translates to $R \sim$ $100,000 \mathrm{~km}(\mathrm{~s} / 1 \mathrm{~cm})$. In reality, however, equal accretion ends before that, when big bodies become oligarchs. Spatial isolation of the big bodies eliminates their mutual accretion and terminates the phase of equal accretion. This occurs when the radial spacing between big bodies $(\Delta a)$ exceeds the size of their individual stirring and feeding zones, $\sim R_{H}$ (Kokubo \& Ida 1998). Writing $2 \pi \Sigma a \Delta a=(4 \pi / 3) \rho R^{3}$, we find

$$
\frac{\Delta a}{R_{H}} \sim \frac{2}{3} \frac{\rho}{\Sigma a} R^{2} \alpha
$$

Inserting eq. (16) into this and setting $\Delta a=R_{H}$, we find the size at which oligarchy sets in is

$$
\begin{aligned}
R_{\text {olig }} & \sim\left(\frac{3 \sigma a}{2 \rho}\right)^{2 / 3} \alpha^{1 / 6} s^{-1 / 3} \\
& \sim 300 \mathrm{~km}\left(\frac{\sigma}{\sigma_{\mathrm{MMKB}}}\right)^{2 / 3}\left(\frac{\mathrm{s}}{1 \mathrm{~cm}}\right)^{-1 / 3},
\end{aligned}
$$

where we have adopted $a=45 A U, \alpha=10^{-4}$ and $\rho=$ $1.5 \mathrm{~g} / \mathrm{cm}^{3}$.

The efficiency of large body formation at this point is (eq. 16)

$$
\frac{\Sigma}{\sigma} \sim 5.5 \%\left(\frac{\sigma}{\sigma_{\mathrm{MMKB}}}\right)^{1 / 3}\left(\frac{s}{1 \mathrm{~cm}}\right)^{-2 / 3} .
$$

And the timescale at this point is (eq. 12)

$$
t_{\text {olig }} \sim 16\left(\frac{\sigma}{\sigma_{\mathrm{MMKB}}}\right)^{1 / 3}\left(\frac{s}{1 \mathrm{~cm}}\right)^{1 / 3} \text { Myrs },
$$

\subsection{End of Oligarchy}

Life continues during oligarchy. Neighboring oligarchs continue to grow simultaneously (and independently) by stirring and accreting their local small bodies (Goldreich et al. 2004), at a rate that is prescribed by eq. (12). As their spheres of influence begin to overlap, they scatter each other to crossing orbits and merge. 
Assuming small bodies do not reach high enough speed to fragment, and that all bodies remain sub-hill (because $\Sigma<\sigma$ and $R \leq s \alpha^{2}$, eqs. 8 \& 11), unity efficiency $(\Sigma \approx \sigma)$ is reached at a time

$$
t_{\mathrm{end}} \sim \frac{\rho s}{\sigma \Omega \alpha} \sim 300\left(\frac{s}{1 \mathrm{~cm}}\right) \mathrm{Myr} .
$$

and at a size

$$
R_{\mathrm{iso}} \sim \sqrt{\frac{\sigma a}{\rho \alpha}} \sim 800 \mathrm{~km}\left(\frac{\sigma}{\sigma_{\mathrm{MMKB}}}\right)^{1 / 2} .
$$

Such a body has the so-called isolation mass, where, moving on a circular orbit, it sweeps up all material within its $R_{H}$ and exhausts its own feed (Lissauer 1987; Kokubo \& Ida 1998; Goldreich et al. 2004). The further growth of isolation mass objects proceeds much more slowly, in the timescale of orbital instabilities.

So the break-down of equal accretion, after the appearance of oligarchs, allows unity efficiency to be reached at a much smaller size than that in eq. (17).

More complicated processes, e.g., small body fragmentation, big body long-range interactions, gap formation, may interfere with the above picture. We defer considerations of these dynamics to future work.

\section{COLLISIONAL GROWTH: SIMULATIONS}

\subsection{Numerical Code and Initial Conditions}

We simulate collisional accretion using a statistical particle-in-a-box code, to compare against the theory presented in $\$ 2$ Our code is described in Shannon et al. (2015), where we showed that various components in the code, including viscous stirring, dynamical friction, accretion, collisional cooling, and collisional fragmentation, perform according to analytical expectations. We also showed that the overall results from this code, running under the collisionless condition, agree with results from previous studies and conform to theoretical expectations.

In the current study, small grains collide frequently. Depending on the impact velocities, collisions can lead to cooling, cratering or catastrophic destruction. We adopt a zero coefficient of restitution (very inelastic collision) for maximum cooling. Catastrophic destruction occurs when the specific kinetic energy in the impact, $0.5\left[M_{1} M_{2} /\left(M_{1}+M_{2}\right)^{2}\right] v^{2}$, with $v$ being the impact velocity, exceeds the disruption threshold, which we adopt from Stewart \& Leinhardt (2009),

$$
\begin{gathered}
Q_{D}^{*} \approx 500\left[\left(\frac{s_{1}}{1 \mathrm{~cm}}\right)^{3}+\left(\frac{s_{2}}{1 \mathrm{~cm}}\right)^{3}\right]^{-1 / 9}\left(\frac{v}{1 \mathrm{~cm} / \mathrm{s}}\right)^{0.8}+ \\
10^{-4}\left[\left(\frac{s_{1}}{1 \mathrm{~cm}}\right)^{3}+\left(\frac{s_{2}}{1 \mathrm{~cm}}\right)^{3}\right]^{0.4}\left(\frac{v}{1 \mathrm{~cm} / \mathrm{s}}\right)^{0.8} \mathrm{erg} / \mathrm{g}
\end{gathered}
$$

where $s_{1}, s_{2}, M_{1}, M_{2}$ are the sizes and masses of the two colliding particles, respectively. This scaling applies for small aggregates that are strength dominated. For an equal-mass impact, this threshold corresponds to an eccentricity of $e \sim 10^{-3}$ at $s=1 \mathrm{~cm}$. Similarly, an $1 \mathrm{~km}$ body should only be destroyed by another $1 \mathrm{~km}$ body at an eccentricity $\sim 10^{-3}$, as they are bound by self-gravity. Speeds this high is not reached until near the end of our simulations. As a result, catastrophic destruction is not a significant process.

On the other hand, cratering (chipping away the target by a low-energy impact) can be important. In the following, we first ignore cratering but return to discuss its effects in 3.4 .

Similar to Shannon et al. (2015), we trace the mass evolution using logarithmic mass bins that are spaced by 0.1 dex. Each bin is characterized by one eccentricity value. The reader is referred to that paper for details on how big bodies are promoted in size, and for our procedure to emulate isolation once oligarchs appear. For simplicity, we consider only a single radial zone, tracking the size spectrum and eccentricity as they evolve in time. Random velocities are assumed to be isotropic $(i \approx e)$, except for big bodies for which we assume $i \ll e$, i.e., they lie in a thin disk (Goldreich et al. 2004).

To simulate the isolation effect of oligarchy, we do not allow the large bodies to accrete each other, once they become oligarchs.

The initial surface density is taken to be $\sigma=\sigma_{\mathrm{MMKB}} \approx$ $0.0016 \mathrm{~g} / \mathrm{cm}^{2}$. We deposit $99.9 \%$ in $s=1 \mathrm{~cm}$ bodies, and the remaining $0.1 \%$ in $1-\mathrm{km}$ bodies. These choices are somewhat arbitrary. Fortunately, our results are little affected if mass in the large seeds is altered by two orders of magnitude up or down.

All bodies are started with an initial eccentricity of $e=10^{-6}$, the Hill velocity of a $1-\mathrm{km}$ body. This is motivated by the argument that, even when one starts from a different condition, a trans-hill state is quickly achieved (Lithwick 2014). This value is also motivated by the fact that the particle disk we consider will be Toomre unstable if the velocity dispersion falls below $e \sim 10^{-6}$. As in Shannon et al. (2015), eccentricity evolution is much faster than mass evolution, and the system relaxes before significant mass evolution takes place.

\subsection{Results}

Results of the collisional growth are plotted in Figure 1 . with Figs. 2 and 3 providing diagnostic details. Overall, the simulation results confirm our analytical picture in $\$ 2$ and we give a brief re-cap below.

Frequent collisions between small grains keep them cold and allow efficient accretion onto km-sized seeds, which are also kept cool by dynamical friction from the small grains. Growth is rapid and within $\sim 10^{5} \mathrm{yrs}$, most seeds have doubled their sizes. The rise in big body population accelerates cannibalism among them and when the big bodies reach sizes $\sim 2 \mathrm{~km}$, smaller than that estimated in eq. (17), we see that equal accretion has set in (Fig. 3).

Defining $R_{\text {peak }}$ to be the size of bodies that dominate stirring (also roughly the largest bodies at any time), we see that as $R_{\text {peak }}$ rises, both the largest bodies and the small grains remain cold, $u, v \leq v_{H}\left(R=R_{\text {peak }}\right.$ ) (Fig. 2), in accordance with eqs. (11) \& (18). Equal accretion predicts that mass in $R \approx R_{\text {peak }}$ rises as $R_{\text {peak }}^{1 / 2}$ (eq. 16), and is indeed observed in the left panel of Fig. 1. By 30 Myrs (eq. 23), oligarchy is reached with $R_{\text {peak }} \sim R_{\text {olig }} \sim$ $300 \mathrm{~km}$, and the efficiency of conglomeration at this point is $\sim 5 \%$, as is estimated in eq. (22).

At this stage, intermediate size bodies are severely depleted, caused by both cannibalism among equal-sized 
bodies and by accretion onto larger bodies. The resultant differential mass spectrum is $d \Sigma / d \ln R \propto R^{2}$, or a differential number distribution of $d N / d R \propto R^{-2}$. This is much more top-heavy compared to the collisionless case where $d N / d R \propto R^{-4}$, and also more so than that obtains assuming a trans-hill collisional growth, $d N / d R \propto R^{-3}$, (Lithwick 2014).

Continuing integration shows that near unity more precisely, $(\approx 25 \%)$ efficiency is reached when $R \sim 800 \mathrm{~km}$ (eq. 25), occurring after $\sim 300$ Myrs of evolution (eq. 24).

\subsection{Collisional vs. Collisionless}

The dividing line between collisional and collisionless regimes lies at $s / R \sim \alpha$ (Lithwick 2014). Throughout our simulations here, with $s / R \sim$ $10^{-5}(\mathrm{~s} / 1 \mathrm{~cm})(R / 1 \mathrm{~km})^{-1} \leq 10^{-5}$, collisional cooling remains important, in contrast to our study in Shannon et al. (2015) where $s \sim 1 \mathrm{~km}$. The different outcomes from these two sets of simulations are summarized in Fig. 4, while readers are referred to Lithwick (2014) for an analytical understanding.

The collisionless growth has an efficiency of $10 \alpha \sim$ $10^{-3}$, while the collisional growth reaches an efficiency of $5 \%$ even at $R=R_{\text {olig. }}$. So to produce the same number of large bodies, the former model requires a disk that is $\sim 100$ times more massive than the collisional one. Moreover, the resultant size spectra differs between the two cases. While the collisionless case deposits equal mass per logarithmic decade, or $d N / d R \propto R^{-4}$, the collisional case leads to a heavy depletion in intermediate sizes, with most of the mass concentrated in the largest bodies, $d N / d R \propto R^{-2}$.

Despite the substantially lower surface density, the collisional case can produce the same $R_{\text {peak }}$ at roughly the same timescale as the collisionless one - because the small bodies' speeds remain small, gravitational focusing aids accretion. Oligarchy, at $R_{\text {peak }} \sim 300 \mathrm{~km}$, is reached in both cases at about 30-60 Myrs.

\subsection{Cratering}

For the material strength law that we adopted (Stewart \& Leinhardt 2009), collisions cause catastrophic destruction between equal-sized bodies at eccentricities $e \gtrsim 10^{-3}$ (for both 1-cm and 1-km bodies; it is smaller for sizes in between), a value reached only near the end of our simulation (see Fig. 1). In contrast, cratering, where a fraction of the target mass is removed, can occur at lower velocities and be potentially important. We study the impact of this process here.

We adopt the approach of Kobayashi \& Tanaka (2010) to write the total ejected mass after a collision as

$$
m_{e}=\frac{\phi}{1+\phi} m,
$$

where $m$ is the target mass, and $\phi$ is the ratio between the specific impact energy to the critical specific energy for catastrophic disruption, $Q_{D}^{*}$. Moreover, we write the mass of the largest fragment as

$$
m_{f}=\frac{\phi}{(1+\phi)^{2}} m .
$$

We deposit the total ejected mass $\left(m_{e}\right)$ as a power law in size between those with masses $m_{f}$ and 0 , with $d N / d R \propto R^{-q}$ and $q=3.5$ (Dohnanyi 1969). So most of the fragment mass lies near $m_{f}$. We place mass for bodies larger than $1 \mu \mathrm{m}$ in the appropriate mass bin and follow them in the numerical integration, while bodies below $1 \mu \mathrm{m}$ are instantaneously removed from the simulation, as would be expected due to radiation pressure from the Sun.

Cratering allows a net mass-loss from the system. While the catastrophic fragmentation of a $1 \mu \mathrm{m}$ grain requires $e \geq 0.01$ (a value barely reachable in our simulation, see Fig. 1), cratering can remove mass at lower velocities.

For the threshold of catastrophic destruction $\left(Q_{D}^{*}\right)$, we adopt the strength formula for small bodies, eq. (26), but weaken it by a factor of 4 , while keeping the threshold for bodies that are gravity-dominated (km-sized and above) unchanged. This factor of 4 is introduced to achieve a good match to observations in \$4.1. We discuss varying this value in 4.3 .

Moreover, to compensate for the fact that much of the initial mass is ground down and blown out, we increase the initial surface density to $\sigma=3 \times \sigma_{\mathrm{MMKB}}$, or $0.3 M_{\oplus}$ in the annulus from 42 to $48 \mathrm{AU}$. This elevated total mass still evades the constraint set by the survival of binary KBOs and the migration stalling of Neptune.

The results are displayed in Fig. 5 . Cratering affects the outcome. Small bodies are now quickly collisionally diminuated. Within a Myr, the cm-grains have largely been removed. Growth of large bodies is initially faster but then is truncated at $R \sim 50 \mathrm{~km}$. At this point, the mass in the big bodies is $\sim 5 \%$ of the total mass. This value is similar to the case without cratering, even though the largest bodies have only reached a fraction of the size of that case. This is because the small body size, $s$, is reduced over time in the cratering simulation (see eq. 16). The form of the size spectrum is similar to that in the fiducial case.

In summary, cratering (and the subsequent blow-out) removes most of the small grains quickly, and arrests the collisional growth at an early stage. It also reduces the overall efficiency of large body formation, to $\sim 5 \%$ in our specific case.

\section{DISCUSSION}

Our work is motivated by the presence of the Cold Classical Kuiper belt, which is hard to explain in the framework of collisionless conglomeration. Here, we compare our simulation results against observations of these bodies. We then proceed to discuss parameter dependence of our models, as well as an alternative formation model, the streaming instability.

\subsection{Matching the cold classical Kuiper belt-Large $\mathrm{KBOs}$}

We first synthesize various surveys to obtain the observed size distribution of large bodies in the Cold Classical belt. We focus on surveys that either distill Cold Classicals from the other populations using dynamical information, or ones that are only sensitive to low inclination objects. The overlapping nature of the transNeptunian populations means that pollution by resonant or scattered populations that happen to lie at low inclinations remains a possibility. For ease of compari- 

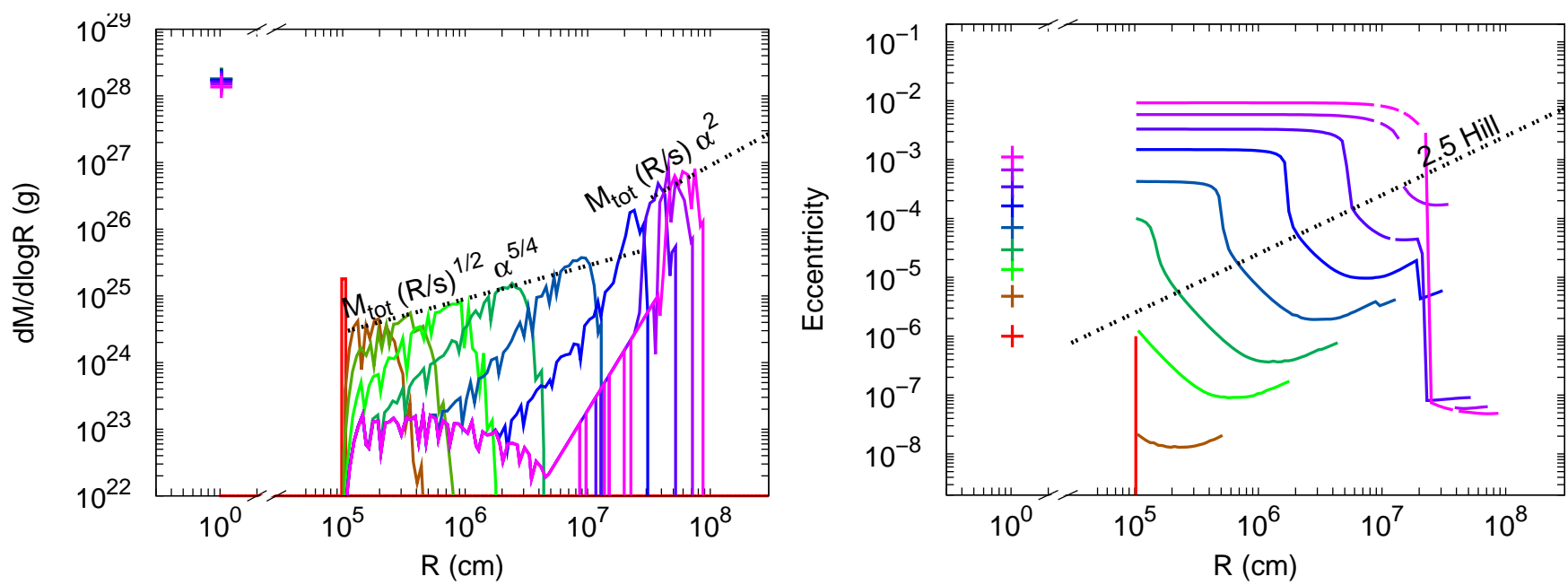

FIG. 1. - Collisional conglomeration in a low-mass disk. The left panel depicts the evolution of the differential mass spectrum, and the right that of eccentricity, both plotted as functions of particle size. Initially, $99.9 \%$ of the mass is in cm-grains and $0.1 \%$ in $\mathrm{km}$-sized rocks. Snapshots of the evolution are taken at 0 (red),1,2,4,8,16,32,64 Myrs, and at 10 Gyrs (magenta line). The lower dashed line shows the predicted mass in the largest bodies as a function of $R$ (eq. 16), valid during equal accretion. At this stage, velocities of both the small and the largest bodies remain subhill (of the largest bodies). Beyond 16 Myrs, the big bodies become oligarchs and they grow by accreting only small bodies (but not each other), in the trans-hill regime (the upper dashed line). There is no collisional cratering and fragmentation included here.

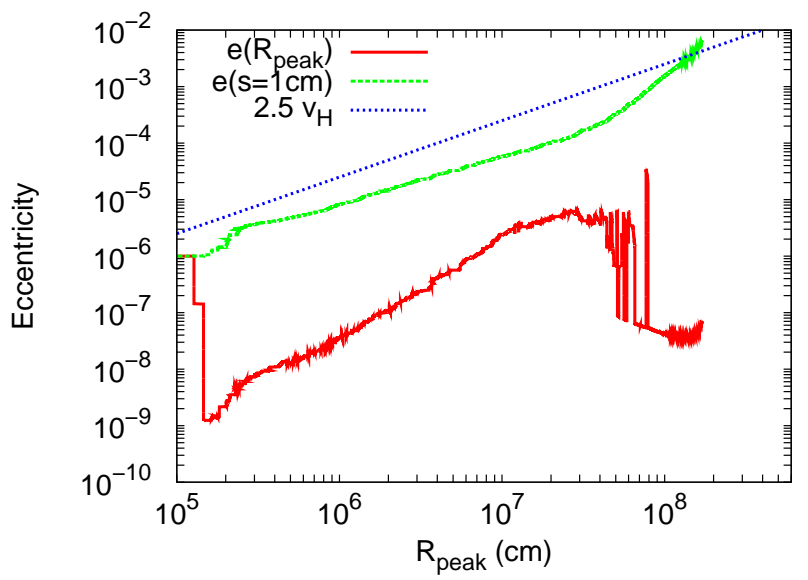

FIG. 2.- Eccentricities for both small grains (green line) and $R_{\text {peak }}$ bodies (red line), as functions of $R_{\text {peak }}$, for the simulation presented in Fig. 1 $R_{\text {peak }}$ is formally the size of large $(\geq 1 \mathrm{~km})$ bodies carrying the most mass, and is roughly the size of the largest bodies at any given time. Before $R_{\text {peak }}$ reaches $\sim 300 \mathrm{~km}$, the evolution is dominated by equal accretion with big and small bodies both remaining sub-hill (compare the blue-dotted and green lines, eq. (18). Once oligarchy sets in at $R_{\text {peak }} \sim 300 \mathrm{~km}$, big-bodies are effectively isolated from each other's stirring and from accreting each other. Their eccentricities drop precipitously and they grow by accreting small bodies only. A trans-hill growth ensues.

son, we convert all observational constraints to differential mass distribution, following Fraser \& Kavelaars (2009) and adopting an albedo of 6\%. For pencil-beam surveys (Bernstein et al. 2004; Schlichting et al. 2009; Fraser et al. |2010), we convert the observed number densities to the total surface densities by assuming a scale height of $5^{\circ}$.

The relevant surveys are: Petit et al. (2011), targeting objects with cold-classical-like orbits with sizes down to $\sim 50 \mathrm{~km}$; Bernstein et al. (2004), a pencil-beam HST survey restricting to objects with $i<5^{\circ}$ and sensitive down to $\sim 10 \mathrm{~km}$; Fraser et al. (2010), similar in selection and sensitivity; and lastly, the serendipitous discov-

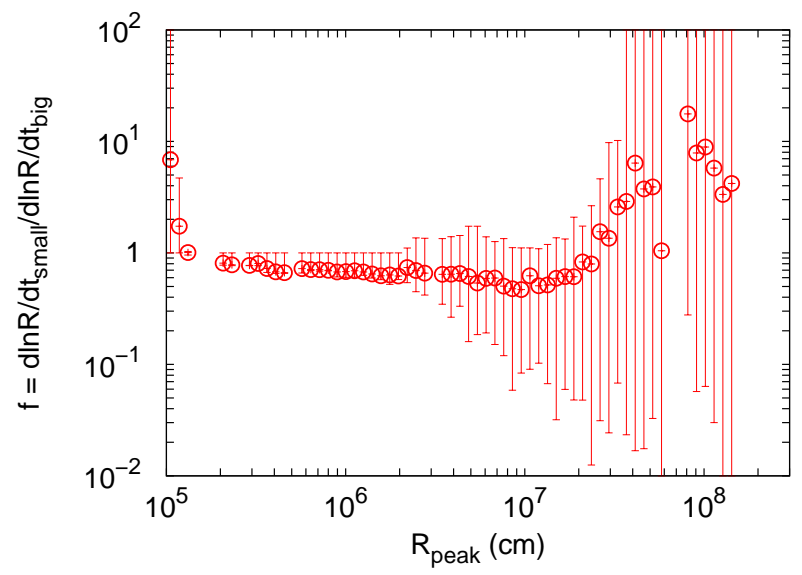

FIG. 3.- Here, we demonstrate equal accretion by plotting the ratio of two accretion rates for bodies of size $R_{\text {peak }}$, that of small body and that of $R \sim R_{\text {peak }}$ bodies. Points are the average ratio at each size, with error bars indicating the highest and the lowest instantaneous values. The system quickly finds the $f \sim 1$ equilibrium (eq. 15). Growth proceeds in equal accretion mode until $R_{\text {peak }}$ reaches $\sim 300 \mathrm{~km}$.

ery of a single $R \sim 0.5 \mathrm{~km}$ object by Schlichting et al. (2009) using stellar occulation - the dynamical class of this object is not well constrained except that it places an upper limit to the Cold Classicals at this size range. A somewhat weaker constraint is published by the TAOs survey (Bianco et al. 2010). Combined together, these surveys indicate that the largest Cold Classicals have radius $\sim 200 \mathrm{~km}$, with the mass peaking around $30 \mathrm{~km}$. The differential mass $(d M / d \log R)$ very roughly falls off as $\sim R^{-2}$ above $30 \mathrm{~km}$ (Petit et al. 2011), and as $\sim R^{1}$ (or possibly steeper) below $30 \mathrm{~km}$ (see Fig. 6).

Collisional growth without cratering (Fig. 1) produce bodies that are too large compared to the observed belt. Cratering simulations, on the other hand, can be easily adapted to reproduce the observed size distribution, as is shown in Fig. 6. With a material strength that is re- 

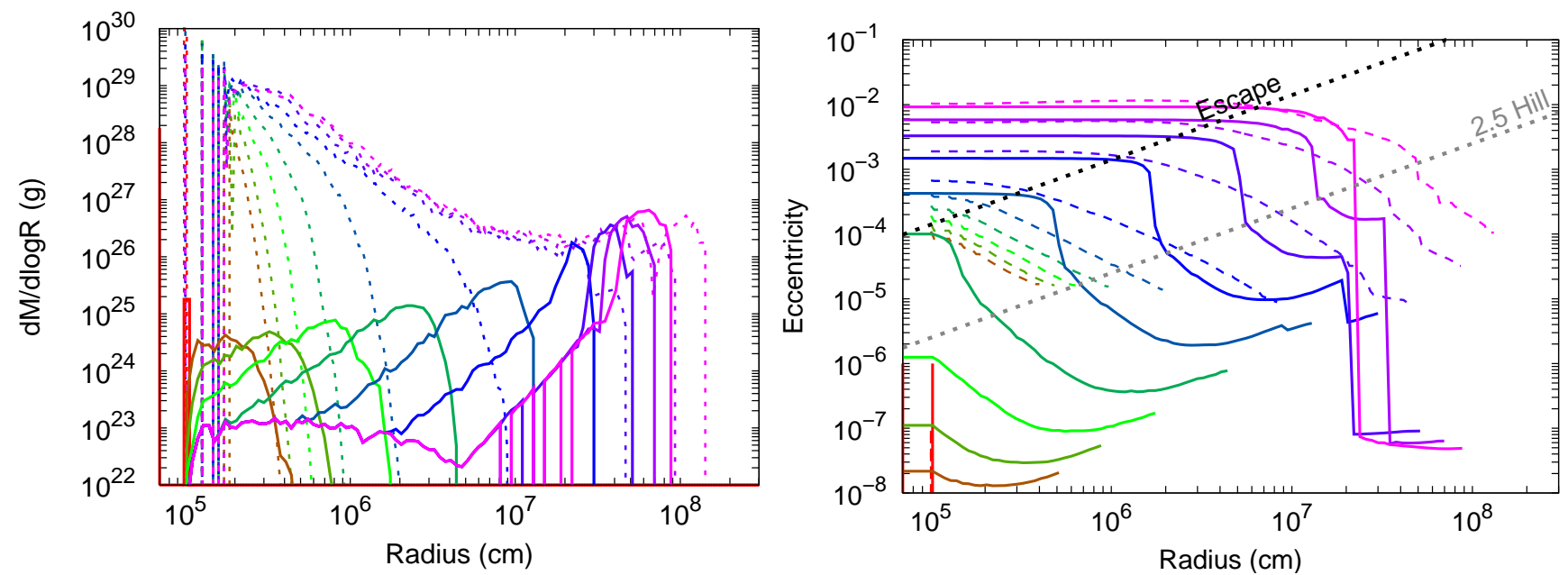

Fig. 4. - A comparison between collisional (solid curves, not showing small grains, Fig. 1 here) and collisionless (dashed curves, Fig. 4 in Shannon et al. (2015)) models of conglomeration. The former case begins with only $1 \%$ mass of the latter. Snapshots for both cases are taken at $0,1,2,4,8,16,32,64,128$ and 256 Myrs. The efficiency of large body formation, measured at late times, is of order unity for the collisional case, and much lower $\left(\sim 10^{-3}\right)$ for the collisionless one, leading to similar numbers of large bodies being produced in both runs. The mass distribution is top-heavy for the collisional one, while flat for the collisionless one.
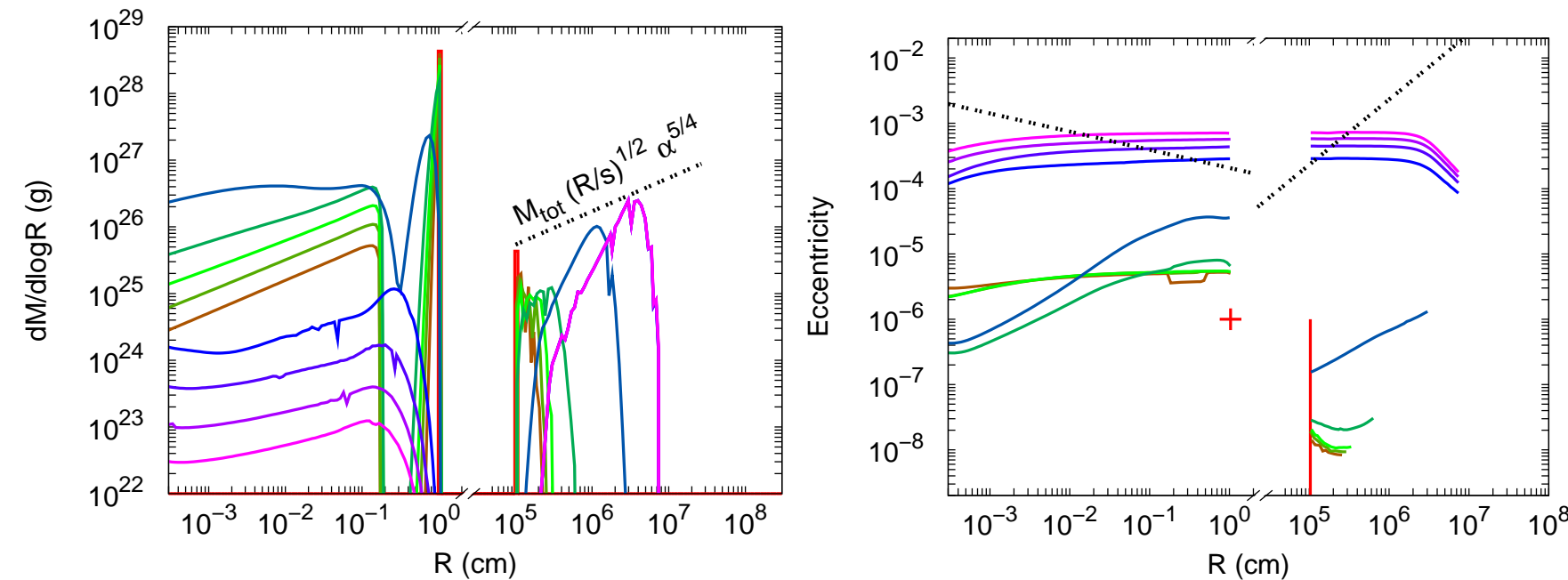

FIG. 5.- Collisional growth, including the effects of cratering. Different curves are snapshots at 0, 0.1, 0.2, 0.4, 0.8, 1.6. 3.2, 6.4, 12.8 and 25.6 Myrs. Growth of large bodies outpaces that in Fig. 1 in the early stage but is stalled after $\sim 2$ Myrs, when the largest bodies reach $\sim 50 \mathrm{~km}$. The stalling occurs because the cm-grains are gradually down-sized and removed from the vicinity. The efficiency at this point is $\sim 5 \%$. The right-hand panel shows the eccentricity evolution, in particular, the dashed curves correspond to our adopted threshold for catastrophic destruction by an equal-mass projectile (eq. 26. reduced by a factor of 4). Cratering is important even well below this threshold due to the presence of smaller projectiles. This is increasingly important in late times when viscous stirring increases the dynamical excitation.

duced from the (Stewart \& Leinhardt 2009) value by a factor of 4 , well within the range of uncertainty, we are able to halt the conglomeration growth and reproduce the observed mass peak at $\sim 30 \mathrm{~km}$. Starting with an initial belt of $0.3 M_{\oplus}$, our simple model reproduces the overall size distribution of Cold Classicals, with a possible exception at very large sizes $(R \geq 100 \mathrm{~km})$.

While cratering is one possible way to arrest growth at intermediate size, it is possible that external heating by a now-absent perturber (or by a more eccentric Neptune) can also do the job. This has the advantage of explaining the current dynamical excitation of the Cold Classicals $(e \sim 0.05$, higher than self-excitation should have produced).

\subsection{A collisional break at $30 \mathrm{~km}$ ?}

The mass fall-off (also called 'roll-over', 'power-law break') observed at below $\sim 30 \mathrm{~km}$ (Bernstein et al. 2004; Fuentes \& Holman 2008; Fraser \& Kavelaars 2009) arises naturally in our growth model. However, in the past, this has instead been interpreted as a collisional break, i.e., bodies below this size have been removed by collisional destructions (Pan \& Sari 2005; Kenvon \& Bromley 2004; Benavidez \& Campo Bagatin 2009; Fraser 2009) 5 However, this interpretation relies on an initially flat mass distribution, $d M / d \log R \propto R^{0}$ (or a size distribution $d N / d R \propto R^{-4}$ ) to have sufficient small bullets to break up the large bodies. Such a mass distribution, however, obtained from collisionless growth

${ }^{5}$ But also see Morbidelli et al. 2009 who attribute it to an observational bias. 


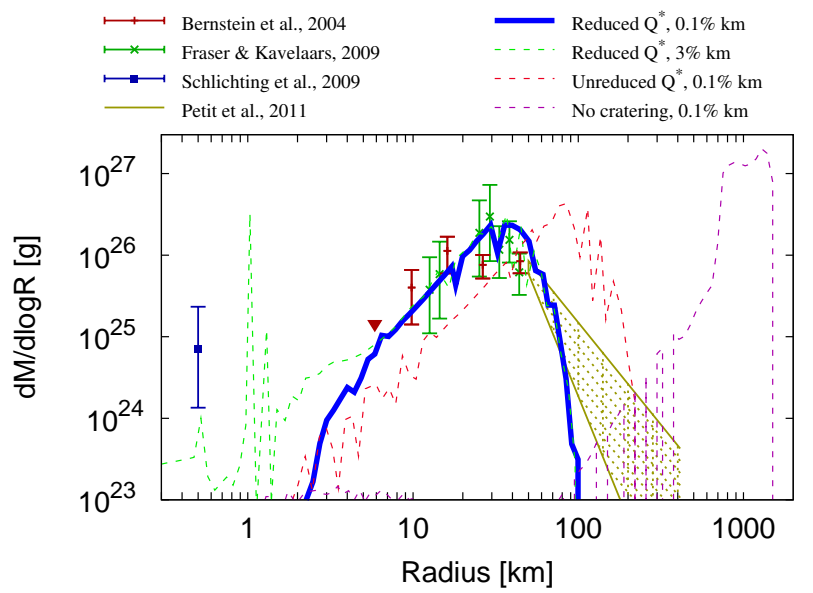

FIG. 6.- Simulated and observed differential mass spectrum for Cold Classical objects. The observed mass distribution of Cold Classicals peak around $\sim 30 \mathrm{~km}$, and fall off on both sides. See text for details of conversion. All four simulations here posit an initial surface density of $\sigma=3 \sigma_{\mathrm{MMKB}}\left(0.3 M_{\oplus}\right.$ within 42 to $\left.48 \mathrm{AU}\right)$. The cratering simulation in Fig. 5 where we reduce the material strength by a factor of 4 from eq. 26 and deposit $0.1 \%$ of the initial mass in km-sized bodies, is shown as solid blue line. Two other cratering simulations, one with more initial mass in the $\mathrm{km}$-bodies $(3 \%)$, the other with unreduced strength, are shown as dotted lines, together with the result from the no-cratering simulation (Fig. 1). The observed mass peak at $\sim 30 \mathrm{~km}$ is replicated in the cratering runs that have reduced strength, while runs with no cratering, or with cratering but at unreduced strength produce bodies that are too large by comparison. Varying the initial mass fraction in $\mathrm{km}$ bodies do not impact the results.

of a very massive disk, is astrophysically unviable (see 1.1.

Intriguingly, a roll-over at roughly the same size has also been observed for Jupiter Trojans (Jewitt et al. 2000; Szabó et al. 2007; Yoshida \& Nakamura 2008) and Neptune Trojans (Sheppard \& Truillo 2010). Their differential mass distributions can all be roughly summarized as $d M / d \log R \propto R^{2}$ at sizes below the peak and $d M / d \log R \propto R^{-2}$ above the peak, a shape also found in the Scattered disk (Vilenius et al. 2012), but with a larger roll-over size $(\sim 300 \mathrm{~km})$. It is difficult to ascribe these features all as collisional breaks, as the physical environments and therefore the collision frequencies are drastically different among these objects. In contrast, a formation model of collisional growth naturally accounts for such a peak. We note that Weidenschilling (2011) has proposed a formation model similar to ours for the asteroid belt, which also exhibits a break at $\sim 50 \mathrm{~km}$.

Integrating the size distribution (e.g., solid line in Fig. 6) forward for 4.5 Gyrs, we find that it suffers little collisional erosion. The expected fractional dust luminosity at current time is $\sim 10^{-8}$, broadly consistent with the upper and lower limits set by Teplitz et al. (1999) and Landgraf et al. (2002).

\subsection{Parameter Dependencies}

We briefly discuss how various model parameters affect the simulation outcome. Much of this discussion follows the analytical scalings in 92

- If we scale up the initial surface density, larger bodies can form.

- If the size of the small grains, $s$, is reduced, we expect a higher formation efficiency faster.

- In contrast, the size of the large seeds, $1 \mathrm{~km}$ in our case, does not much influence the outcome.

- Similarly, the initial mass fraction in these large seeds makes little difference to the final size spectrum, as is seen in Fig. 6, as long as these seeds remain a minor component.

- If these seeds are totally absent, however, the initial growth will have to rely on the uncertain process of particle sticking, and a different growth scenario may occur (Lithwick 2014; Kenyon \& Bromley 2015).

- If the material strength, relevant for the outcome of cratering collisions, is decreased, small bodies can be more easily destroyed, leading to a shortened growth episode and a smaller maximum size (Fig. 6). Conversely, if material strength is stronger than assumed here, the maximum size increases. This would be necessary if the mass peak is around $70 \mathrm{~km}$ as found by Fraser et al. (2014).

\subsection{Alternative Formation Theories}

Is it possible that large Kuiper belt objects form via other routes, as opposed to the gradual conglomeration as investigated here? Alternatives include the direct collapse of a self-gravitating disk (Toomre 1964; Goldreich \& Ward 1973); incremental growth by direct sticking of small grains (e.g. Weidenschilling 1980; Dullemond \& Dominik 2005; Windmark et al. 2012); the streaming instability in a gas-solid coupled fluid (Youdin \& Goodman 2005; Johansen et al. 2007); abnormal concentration of dust grains by turbulence (Cuzzi et al. 2010); and the accretion of 'pebbles' (cmsized particles) by larger planetesimals in a gas-rich disk (Lambrechts \& Johansen 2012).

The first option, the Toomre instability, could be important if the disk initially contained only small grains. If the grains are able to avoid stirring by gas turbulence of the type argued by Weidenschilling (1995) and continue to cool below $e=10^{-6}$ to reach Toomre number $Q=1$, preliminary calculations (Michikoshi et al. 2007, 2009) indicate that some Toomre mass scale bodies may form. These are of order $\mathrm{km}$ in size for our parameters. This then returns us to exactly the scenario we posit here. So the Toomre instability may provide the backdrop for our work.

In a gaseous nebula, direct sticking of small grains may not be efficient in producing ever larger bodies, even though naive calculations (perfect sticking) predict otherwise. This is because as bodies grow, collisions may become increasingly destructive and be dominated by fragmentation (Dullemond \& Dominik 2005; Brauer et al. 2008).

What about the popular streaming instability? If the primordial Kuiper belt, when gas was present, was depleted in solid materials relative to that of MMSN, the streaming instability may be difficult to initiate. If it was of MMSN abundance, on the other hand, then it needs to convert only $\sim 0.1 \%$ of the initial solid into large bodies. It is not clear if this is a natural outcome of the streaming instability - although to be fair, our mechanism here 
started from an initial condition that we have also not yet justified. We note that there are recent attempts to predict the size distribution resulting from the streaming instability (Johansen et al. 2015; Simon et al. 2015) and more work is needed to quantitatively compare these against the observed Cold Classical objects.

Cuzzi et al. (2010) has proposed that small grains in a turbulent gas disk may be concentrated at certain regions to such a high density as to be self-gravitationally unstable. This proposal requires more physical understanding of the turbulence than is currently available.

'Pebble accretion' in a gas disk, on the other hand, shares some similar attributes to the model presented here. While accretion in our case is aided by the low dynamical excitation of cm-sized bodies as a result of frequent collisions, accretion in that scenario is aided by gas drag on $\mathrm{cm}$-sized bodies. The size distribution arising from 'pebble accretion' is not yet investigated and is an interesting future direction.

In summary, alternative theories are available but are not yet investigated in sufficient detail to be tested.

\section{SUMMARY}

The consensus is building that the Cold Classical Kuiper Belt objects likely formed in situ and have not suffered much dynamical turmoil from the giant planets. They are reliable fossil records for deciphering the environment and the mode of their formation.

It is often thought that the current-day anemic Kuiper belt is but a skeletal remain of the primordial massive disk, given that models of collisionless conglomeration requires a massive disk to form large KBOs quickly and in sufficient numbers. Moreover, it has been argued that the observed 'roll-over' at size $R \sim 30 \mathrm{~km}$ is a result of collisional diminuation over the past few billion years. In this study, we challenge both these ideas. We argue that the Cold Classicals may have formed with high efficiency, out of a low-mass disk that is only a few percent of the MMSN value, and that the size break at $30 \mathrm{~km}$ is primordial and may not be related to collisional erosion.

In our model, much of the primordial mass is in small grains that frequently collide and cool. This allows large KBOs to form quickly in a low-density environment. The efficiency of large-body formation can reach order unity, if growth continues undisturbed. The resultant mass distribution of large KBOs is top-heavy with $d M / d \log R \propto R^{2}$, in contrast with the flat mass distribution from collisionless growth.

Even in this low mass disk, conglomeration can, within a few million years, convert almost all mass into bodies of size $\sim 1000 \mathrm{~km}$. To reproduce the observed peak at $\sim 30 \mathrm{~km}$, we introduce cratering collisions. We show that, for plausible material strengths, growth of large bodies can be arrested at an early stage, as small grains can be demolished and removed from the system. Alternatively, growth could also be arrested by, e.g., external excitation by nearby planets (e.g., Thommes et al. 1999; Tsiganis et al.|2005), or a stellar flyby in the denser birth environment (Kobayashi et al. 2005; Melita et al. 2005).

Processes not considered in our simple model include gas damping (Rafikov 2003a), gas dynamical friction (Grishin \& Perets 2015), semi-collisional accretion (Schlichting \& Sari 2007), and the influence of KBO binarity on accretion. Each could significantly affect the outcome of our model.

In conclusion, we suggest that Cold Classical KBOs likely formed not out of a MMSN of solid material, but out of a 'minimum mass Kuiper belt' (MMKB), one that is but $\sim 1 \%$ of the MMSN surface density. This belt, with its low density, may well represent the true outer edge of the Solar System. This edge could explain why Neptune's outward migration is stalled at $30 \mathrm{au}$, and we might therefore expect no more large bodies to be formed beyond the Cold Classical region.

We thank Eugene Chiang, Alexander Krivov, Chris Ormel and Hilke Schlichting for helpful discussions. We are also grateful for an anonymous referee who compels us to examine our assumptions closely. YW acknowledges grants from NSERC. YL acknowledges grants AST-1109776 and AST-1352369 from NSF, and NNX14AD21G from NASA. AS was supported by the government of Ontario by a Ontario Graduate Scholarship in Science and Technology; and is supported by the European Union through ERC grant number 279973.

\section{REFERENCES}

Barucci, M. A., Boehnhardt, H., Cruikshank, D. P., Morbidelli, A., \& Dotson, R. 2008, The Solar System Beyond Neptune Benavidez, P. G. \& Campo Bagatin, A. 2009, Planet. Space Sci., 57, 201

Bernstein, G. M., Trilling, D. E., Allen, R. L., Brown, M. E., Holman, M., \& Malhotra, R. 2004, AJ, 128, 1364

Bianco, F. B., Zhang, Z., Lehner, M. J., Mondal, S., King, S., Giammarco, J., Holman, M. J., Coehlo, N. K., Wang, J., Alcock, C., Axelrod, T., Byun, Y., Chen, W. P., Cook, K. H., Dave, R., de Pater, I., Kim, D., Lee, T., Lin, H., Lissauer, J. J. Marshall, S. L., Protopapas, P., Rice, J. A., Schwamb, M. E., Wang, S., \& Wen, C. 2010, AJ, 139, 1499

Blum, J. \& Wurm, G. 2008, ARA\&A, 46, 21

Brauer, F., Dullemond, C. P., \& Henning, T. 2008, A\&A, 480, 859

Brown, M. E. 2001, AJ, 121, 2804

Brucker, M. J., Grundy, W. M., Stansberry, J. A., Spencer, J. R., Sheppard, S. S., Chiang, E. I., \& Buie, M. W. 2009, Icarus, 201, 284
Bryden, G., Beichman, C. A., Trilling, D. E., Rieke, G. H., Holmes, E. K., Lawler, S. M., Stapelfeldt, K. R., Werner, M. W., Gautier, T. N., Blaylock, M., Gordon, K. D., Stansberry, J. A., \& Su, K. Y. L. 2006, ApJ, 636, 1098

Cuzzi, J. N., Hogan, R. C., \& Bottke, W. F. 2010, Icarus, 208, 518 Dawson, R. I. \& Murray-Clay, R. 2012, ApJ, 750, 43

Dohnanyi, J. W. 1969, J. Geophys. Res., 74, 2531

Dullemond, C. P. \& Dominik, C. 2005, A\&A, 434, 971

Edgeworth, K. E. 1949, MNRAS, 109, 600

Elliot, J. L., Kern, S. D., Clancy, K. B., Gulbis, A. A. S., Millis, R. L., Buie, M. W., Wasserman, L. H., Chiang, E. I., Jordan, A. B., Trilling, D. E., \& Meech, K. J. 2005, AJ, 129, 1117

Fernandez, J. A. \& Ip, W.-H. 1984, Icarus, 58, 109

Fraser, W. \& Brown, M. E. 2011, in Bulletin of the American Astronomical Society, Vol. 43, American Astronomical Society Meeting Abstracts \#217, \#306.04-+

Fraser, W. C. 2009, ApJ, 706, 119

Fraser, W. C., Brown, M. E., Morbidelli, A., Parker, A., \& Batygin, K. 2014, ApJ, 782, 100

Fraser, W. C., Brown, M. E., \& Schwamb, M. E. 2010, Icarus, 210,944 
Fraser, W. C. \& Kavelaars, J. J. 2009, AJ, 137, 72

Fuentes, C. I. \& Holman, M. J. 2008, AJ, 136, 83

Goldreich, P., Lithwick, Y., \& Sari, R. 2004, ARA\&A, 42, 549

Goldreich, P. \& Ward, W. R. 1973, ApJ, 183, 1051

Gomes, R. S., Fernández, J. A., Gallardo, T., \& Brunini, A. The Scattered Disk: Origins, Dynamics, and End States, ed.

Barucci, M. A., Boehnhardt, H., Cruikshank, D. P., Morbidelli, A., \& Dotson, R., 259-273

Gomes, R. S., Morbidelli, A., \& Levison, H. F. 2004, Icarus, 170, 492

Greenberg, R., Hartmann, W. K., Chapman, C. R., \& Wacker, J. F. 1978, Icarus, 35, 1

Grishin, E. \& Perets, H. B. 2015, ArXiv e-prints

Hayashi, C. 1981, Progress of Theoretical Physics Supplement, 70,35

Ida, S. \& Makino, J. 1992, Icarus, 96, 107

Isella, A., Pérez, L. M., \& Carpenter, J. M. 2012, ApJ, 747, 136

Jewitt, D. \& Luu, J. 1993, Nature, 362, 730

Jewitt, D. C., Trujillo, C. A., \& Luu, J. X. 2000, AJ, 120, 1140

Johansen, A., Mac Low, M.-M., Lacerda, P., \& Bizzarro, M. 2015, Science Advances, 1, 15109

Johansen, A., Oishi, J. S., Low, M.-M. M., Klahr, H., Henning, T., \& Youdin, A. 2007, Nature, 448, 1022

Kelley, M. S., Lindler, D. J., Bodewits, D., A'Hearn, M. F., Lisse, C. M., Kolokolova, L., Kissel, J., \& Hermalyn, B. 2013, Icarus, 222,634

Kenyon, S. J. \& Bromley, B. C. 2004, AJ, 128, 1916

- 2008, ApJS, 179, 451

-. 2015, ApJ, 806, 42

Kenyon, S. J. \& Luu, J. X. 1998, AJ, 115, 2136

-. 1999, ApJ, 526, 465

Kobayashi, H., Ida, S., \& Tanaka, H. 2005, Icarus, 177, 246

Kobayashi, H. \& Tanaka, H. 2010, Icarus, 206, 735

Kokubo, E. \& Ida, S. 1998, Icarus, 131, 171

Kretke, K. A. \& Levison, H. F. 2015, Icarus, 262, 9

Lacerda, P., Fornasier, S., Lellouch, E., Kiss, C., Vilenius, E., Santos-Sanz, P., Rengel, M., Müller, T., Stansberry, J., Duffard, R., Delsanti, A., \& Guilbert-Lepoutre, A. 2014, ApJ, 793, L2

Lambrechts, M. \& Johansen, A. 2012, A\&A, 544, A32

Landgraf, M., Liou, J.-C., Zook, H. A., \& Grün, E. 2002, AJ, 123, 2857

Levison, H. F., Morbidelli, A., Vanlaerhoven, C., Gomes, R., \& Tsiganis, K. 2008, Icarus, 196, 258

Levison, H. F. \& Stern, S. A. 2001, AJ, 121, 1730

Lissauer, J. J. 1987, Icarus, 69, 249

Lithwick, Y. 2014, ApJ, 780, 22

Luu, J., Marsden, B. G., Jewitt, D., Trujillo, C. A., Hergenrother, C. W., Chen, J., \& Offutt, W. B. 1997, Nature, 387, 573

Malhotra, R. 1993, Nature, 365, 819

-. 1995, AJ, 110, 420

Massironi, M. \& Simioni, E. and. Marzari, F. a. C. G. a. L. a. P. M. a. J. L. a. N. G. a. L. S. a. E. M. R. a. P. F. a. S. F. 2015 , Nature

Melita, M. D., Larwood, J. D., \& Williams, I. P. 2005, Icarus, 173,559

Michikoshi, S., Inutsuka, S.-i., Kokubo, E., \& Furuya, I. 2007, ApJ, 657, 521

Michikoshi, S., Kokubo, E., \& Inutsuka, S.-i. 2009, ApJ, 703, 1363

Morbidelli, A., Gaspar, H. S., \& Nesvorny, D. 2014, Icarus, 232, 81

Morbidelli, A., Levison, H. F., Bottke, W. F., Dones, L., \& Nesvorný, D. 2009, Icarus, 202, 310

Noll, K. S., Grundy, W. M., Stephens, D. C., Levison, H. F., \& Kern, S. D. 2008, Icarus, 194, 758

Ormel, C. W., Dullemond, C. P., \& Spaans, M. 2010, Icarus, 210, 507

Ormel, C. W. \& Klahr, H. H. 2010, A\&A, 520, A43
Pan, M. \& Sari, R. 2005, Icarus, 173, 342

Parker, A. H. \& Kavelaars, J. J. 2010, ApJ, 722, L204

—. 2012, ApJ, 744, 139

Parker, A. H., Kavelaars, J. J., Petit, J.-M., Jones, L., Gladman, B., \& Parker, J. 2011, ApJ, 743, 1

Pérez, L. M., Carpenter, J. M., Chandler, C. J., Isella, A., Andrews, S. M., Ricci, L., Calvet, N., Corder, S. A., Deller, A. T., Dullemond, C. P., Greaves, J. S., Harris, R. J., Henning, T., Kwon, W., Lazio, J., Linz, H., Mundy, L. G., Sargent, A. I., Storm, S., Testi, L., \& Wilner, D. J. 2012, ApJ, 760, L17

Perna, D., Barucci, M. A., Fornasier, S., DeMeo, F. E., Alvarez-Candal, A., Merlin, F., Dotto, E., Doressoundiram, A., \& de Bergh, C. 2010, A\&A, 510, A53+

Petit, J.-M., Kavelaars, J. J., Gladman, B. J., Jones, R. L., Parker, J. W., Van Laerhoven, C., Nicholson, P., Mars, G., Rousselot, P., Mousis, O., Marsden, B., Bieryla, A., Taylor, M., Ashby, M. L. N., Benavidez, P., Campo Bagatin, A., \& Bernabeu, G. 2011, AJ, 142, 131

Rafikov, R. R. 2003a, AJ, 126, 2529

-. 2003b, AJ, 125, 942

Safronov, V. S. 1969, Evoliutsiia doplanetnogo oblaka., ed. Safronov, V. S.

Schlichting, H. E., Ofek, E. O., Wenz, M., Sari, R., Gal-Yam, A., Livio, M., Nelan, E., \& Zucker, S. 2009, Nature, 462, 895

Schlichting, H. E. \& Sari, R. 2007, ApJ, 658, 593

—. 2011, ApJ, 728, 68

Shannon, A. \& Wu, Y. 2011, ApJ, 739, 36

Shannon, A., Wu, Y., \& Lithwick, Y. 2015, ApJ, 801, 15

Sheppard, S. S. \& Trujillo, C. A. 2010, ApJ, 723, L233

Simon, J. B., Armitage, P. J., Li, R., \& Youdin, A. N. 2015, ArXiv e-prints

Stewart, S. T. \& Leinhardt, Z. M. 2009, ApJ, 691, L133

Szabó, G. M., Ivezić, Ž., Jurić, M., \& Lupton, R. 2007, MNRAS, 377, 1393

Tegler, S. C. \& Romanishin, W. 2000, Nature, 407, 979

Teplitz, V. L., Stern, S. A., Anderson, J. D., Rosenbaum, D. Scalise, R. J., \& Wentzler, P. 1999, ApJ, 516, 425

Thommes, E. W., Duncan, M. J., \& Levison, H. F. 1999, Nature, 402,635

Toomre, A. 1964, ApJ, 139, 1217

Trotta, F., Testi, L., Natta, A., Isella, A., \& Ricci, L. 2013, A\&A, 558, A64

Trujillo, C. A. \& Brown, M. E. 2002, ApJ, 566, L125

Tsiganis, K., Gomes, R., Morbidelli, A., \& Levison, H. F. 2005, Nature, 435, 459

Vilenius, E., Kiss, C., Mommert, M., Müller, T., Santos-Sanz, P., Pal, A., Stansberry, J., Mueller, M., Peixinho, N., Fornasier, S. Lellouch, E., Delsanti, A., Thirouin, A., Ortiz, J. L., Duffard, R., Perna, D., Szalai, N., Protopapa, S., Henry, F., Hestroffer, D., Rengel, M., Dotto, E., \& Hartogh, P. 2012, A\&A, 541, A94

Vitense, C., Krivov, A. V., \& Löhne, T. 2010, A\&A, 520, A32+

Weidenschilling, S. J. 1977a, MNRAS, 180, 57

-. 1977b, Ap\&SS, 51, 153

-. 1980, Icarus, 44, 172

-. 1995, Icarus, 116, 433

-. 2011, Icarus, 214, 671

Weidenschilling, S. J., Spaute, D., Davis, D. R., Marzari, F., \& Ohtsuki, K. 1997, Icarus, 128, 429

Wetherill, G. W. \& Stewart, G. R. 1989, Icarus, 77, 330

Windmark, F., Birnstiel, T., Güttler, C., Blum, J., Dullemond, C. P., \& Henning, T. 2012, A\&A, 540, A73

Yoshida, F. \& Nakamura, T. 2008, PASJ, 60, 297

Youdin, A. N. \& Goodman, J. 2005, ApJ, 620, 459

Zsom, A., Ormel, C. W., Güttler, C., Blum, J., \& Dullemond, C. P. 2010, A\&A, 513, A57 